\title{
Central nervous system filariasis masquerading as a glioma: case report
}

\author{
Adesh Shrivastava, MCh, ${ }^{1}$ Prateek Arora, MBBS, ${ }^{1}$ Akriti Khare, MD, ${ }^{2}$ Garima Goel, MD, ${ }^{2}$ and \\ Neelkamal Kapoor, MD²
}

\begin{abstract}
Departments of ${ }^{1}$ Neurosurgery and ${ }^{2}$ Pathology, All India Institute of Medical Sciences, Saket Nagar, Bhopal, Madhya Pradesh, India
\end{abstract}

\begin{abstract}
Filariasis, an endemic zoonosis in the Southeast Asia region, has been reported to affect various organs as well as the central nervous system (CNS). Inflammatory reactions mimicking those from neoplastic lesions clinically and radiologically have been reported in the breast and urinary bladder. To date, a CNS manifestation of filarial infestation has been reported in the form of meningoencephalitis. The authors here present an interesting case of a young man presenting in status epilepticus, which on radiological evaluation appeared to be a glioma. However, postoperative histopathological examination changed the provisional diagnosis to a filarial infection of the CNS mimicking a primary CNS neoplasm.

https://thejns.org/doi/abs/10.3171/2016.9.JNS161092
\end{abstract}

KEY WORDS cerebral filariasis; glioma; gliosis; infection

$\mathrm{M}$ ICROFILARIAL infestations are common in the Southeast Asia region and a major health problem in India. The disease usually affects the lower limbs, spermatic cord, and epididymis, whereas involvement of the breast, thyroid, body fluids, skin, or oral cavity is unusual. ${ }^{7}$ Microfilariae of Wuchereria bancrofti, Loa loa, Dirofilaria, and Onchocerca volvulus have been identified in the central nervous system (CNS). ${ }^{2}$ Here, we report an interesting and unusual case of a young man who presented in an acute setting with seizures and a large intraaxial lesion in the left parietal lobe, appearing to be a neoplastic lesion on CT scanning; however, upon further evaluation and histopathological analysis the diagnosis was filariasis-induced gliosis.

\section{Case Report}

History and Examination

A 27-year-old man presented to neurosurgical emergency in status epilepticus. He had multiple episodes of generalized tonic-clonic seizures, which were acute in onset and a day in duration. There was no previous history of seizures, headaches, or neurological deficits. Plain CT and contrast MRI of the brain revealed a large, intraaxial space-occupying lesion $6 \times 5 \mathrm{~cm}$ in size in the left temporoparietal lobe, with surrounding diffuse cerebral edema causing midline shift toward the right (Fig. 1).

\section{Operation}

With a provisional diagnosis of left parietal glioma, we performed an emergency craniotomy with decompression. The mass was soft, partly "suckable," and amenable to cavitron ultrasonic surgical aspiration with areas of rubbery consistency somewhat similar to an intermediate-grade glioma on gross appearance.

Histopathological examination of the specimen revealed brain parenchyma with diffuse infiltration of pleomorphic gemistocytes showing nuclear hyperchromasia and elongation. There was perivascular cuffing of lymphocytes and focal microcyst formation. Mitoses were extremely sparse (0-1 mitotic figure/50 hpf). Multiple microfilariae with nuclei extending up to the tip were seen embedded amid the gemistocytes and blood vessel wall (Fig. 2). A peripheral smear done thereafter also showed microfilariae; however, the absolute eosinophil count was within normal limits. A course of diethylcarbamazine (DEC) was completed, and microfilariae from the peripheral blood smear cleared.

A provisional report of Grade II gemistocytic astrocytoma was made, and immunohistochemical (IHC) analysis with IDH1, p53, GFAP, and MIB-1 was advised for confirmation of the diagnosis and to rule out reactive gliosis in view of the presence of microfilariae in the brain parenchyma as well as the peripheral blood. Subsequently, IHC markers for GFAP showed background positivity suggestive of reactive gliosis. This was further supported by an increased cellularity of astrocytes with frequent gemistocytes, infrequent mitoses, and only mild atypia (Fig. 2).

\section{Postoperative Course}

The patient had an uneventful recovery period and be- 

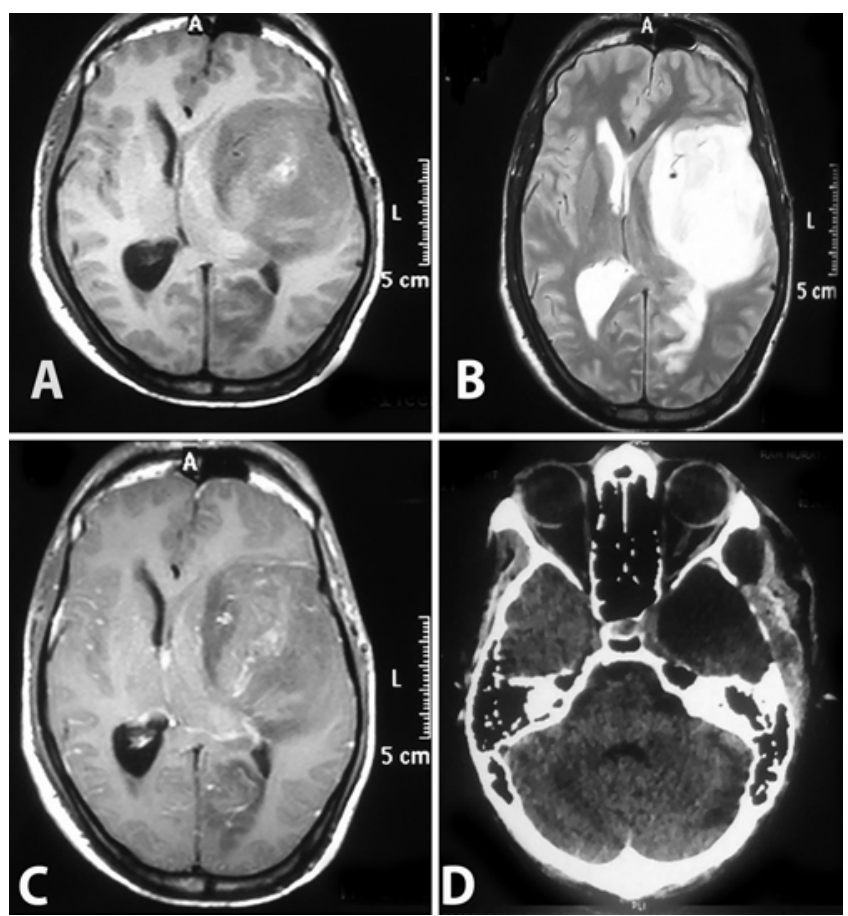

FIG. 1. Preoperative axial T1-weighted (A), T2-weighted (B), and gadolinium-enhanced Tl-weighted (C) MR images showing a mass lesion. Postoperative CT scan showing complete resolution of the lesion after surgery and a course of the anthelmintic drug DEC (D).

came symptom free. Repeat scanning at 6 months showed no signs of residual or recurrent disease.

\section{Discussion}

Filariasis affects over 120 million people worldwide and is endemic to 80 countries. One of 3 nematodesW. bancrofti, Brugia malayi, and Brugia timori-causes lymphatic filariasis, while L. loa causes filarial infection of subcutaneous tissues. The larvae of these worms are the microfilariae, which are transmitted by mosquitoes to humans, in whom they can be found in the blood and show periodicity. ${ }^{6}$ Neurological manifestation of filarial infestation is rare; however, encephalitis following treatment with DEC in patients with $L$. loa filariasis has been reported. ${ }^{3}$ Acute disseminated meningoencephalitis following $W$. bancrofti infection has been reported as well. ${ }^{8}$

The neuropathological changes in parasitic infestation of the CNS vary, depending on factors such as type and size of the parasite, geographic strain variations in virulence factors, and differences in the host immune response. Congestion of the leptomeninges, cerebral edema, thrombosis, hemorrhage, vasculitis, necrosis, calcification, abscesses, meningeal and perivascular polymorphonuclear and mononuclear inflammatory infiltrates, microglial nodules, gliosis, granulomas, and fibrosis can be found. ${ }^{9}$ On reviewing the literature on the detection of microfilaria with a CNS neoplasm, we noted that Aron et al. reported microfilaria in the cyst fluid of tumors of the brain and that Singh et al. reported microfilaria in a squash preparation of oligodendroglioma, but no microfilariae were identified in the histological sections in either of these 2 cases. ${ }^{2,11}$
Among the CNS neoplasm histopathological examinations performed, microfilariae have been demonstrated in only 1 case of hemangioblastoma of the cerebellum. ${ }^{1}$ To date, there have been no reports of cerebral filariasis presenting as a space-occupying lesion either clinically or radiologically.

Distinguishing low-grade glioma (astrocytomas) from reactive gliosis is a major challenge in the routine practice of surgical neuropathology. ${ }^{12}$ Gliosis is a reaction of the CNS to any injury. It reflects the specific ability of astrocytes to respond to CNS injury and disease. ${ }^{10}$ In gliosis, astrocytes show more uniform spacing rather than occurring at the margins in glioma. The nuclear cytoplasmic ratio of reactive astrocytes is less than that of neoplastic astrocytes, which show atypia. ${ }^{12}$ Gliosis is typically polymorphic as reactive astrocytes are rarely in the same stage of reactivity at one time and thus show a mixture of astrocytes, some with enlarged nuclei and frequent gemistocytes. Reactive astrocytes exhibit prominent cytoplasmic processes, and mitoses are scarce or absent. In contrast, glioma has a monomorphic appearance containing densely packed tumor glial cells extending few cytoplasmic processes. Moreover, the neoplastic astrocytes are located in the proximity of apparently normal or proliferating vessels; mitoses are numerous, and neoplastic astrocytes show atypical, irregular, elongated, heterochromatic nuclei. ${ }^{12}$ Gemistocytic astrocytoma is a variant of diffuse astrocytoma in which gemistocytes amount to more than approximately $20 \%$ of tumor cells. Our case also showed frequent gemistocytes; thus, our differential diagnoses were gemistocytic astrocytoma (WHO Grade II) and gliosis. Because gemistocytes are frequently seen in response to injury, gemistocytic cells in an astrocytoma must be distinguished from reactive astrocytes. The nuclei of reactive astrocytes, although enlarged, are not atypical, hyperchromatic, or pleomorphic. On IHC analysis with GFAP, gliosis shows abundant GFAP-positive reactive astrocytes, whereas tumoral astrocytes associated with astrogliomas show various degrees of cytoplasmic reactivity for GFAP and additionally display nuclear atypia. Glial fibrillary acidic protein is regarded much like an astrocytic common denominator because of its presence in normal and reactive astrocytes, as well as in tumor cells. Consequently, it is considered to have diagnostic limitations. However, a recent report has demonstrated differences in the expression of GFAP in all 3 astrocytic phenotypes (normal, reactive, and tumoral). ${ }^{12}$ In the present case there was an increased cellularity of astrocytes with frequent gemistocytes, infrequent mitoses, and minimal atypia.

With the differential diagnoses of gemistocytic astrocytoma versus gliosis, we advised IHC with GFAP, which showed diffuse positivity with positive cytoplasmic processes. Thus, the pattern of GFAP resembled reactive astrogliosis, as mentioned in an earlier study..$^{12}$ Although GFAP expression has limited value in differentiating reactive gliosis from low-grade glioma, looking into the pattern of GFAP expression and the clinical findings led us to a final diagnosis of gliosis in response to microfilarial infestation. As in this case, filariasis has mimicked a neoplastic lesion in other organs as well, namely the breast and urinary bladder. ${ }^{4,5}$ 

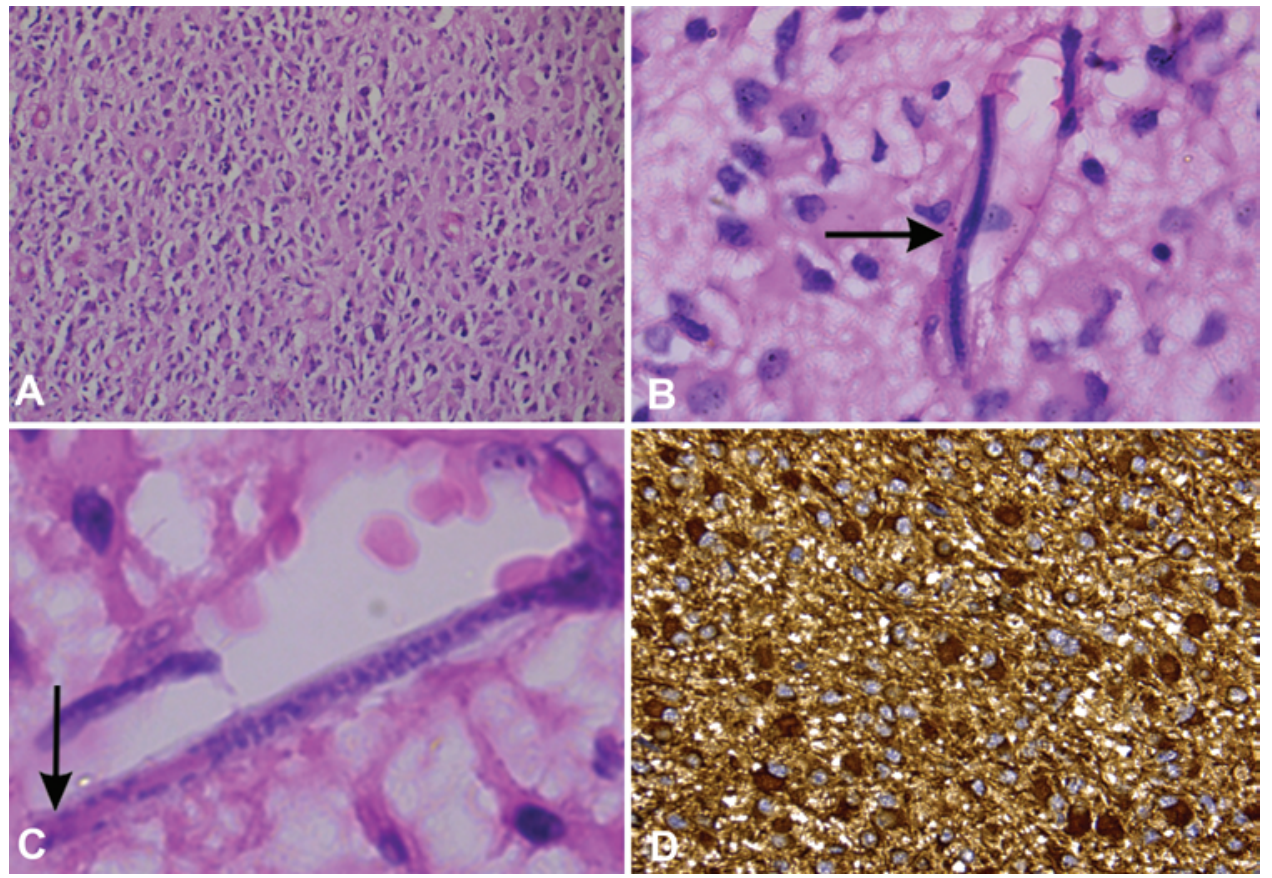

FIG. 2. A: Diffuse proliferation of reactive gemistocytes and astrocytes along with congested capillaries. B: Intravascular microfilaria (arrow) eliciting reactive gemistocytic proliferation in the surrounding tissue. C: Enlarged view of microfilaria with nuclei extending to the tip (arrow). D: Gemistocytes and astrocytes showing diffuse positivity for GFAP with distinct cytoplasmic processes. $H \& E(A-C)$ and DAB (D), original magnification $\times 200(A), \times 1000(B$ and C), and $\times 400(D)$. Figure is available in color online only.

\section{Conclusions}

Cerebral filariasis has been previously reported but not as a space-occupying lesion in the brain. Filarial inflammatory mass has been reported in other organs (urinary bladder and breast), clinically and radiologically appearing as neoplastic lesions. This case is the first report describing a similar picture in the CNS. And this infective lesion had a very good prognosis for the patient as compared with the prognosis for a glioma of similar proportions. Therefore, in endemic areas with a high suspicion for filariasis, this differential diagnosis should be considered a possibility.

\section{References}

1. Agarwal PK, Srivastava AN, Agarwal N: Microfilariae in association with neoplasms. Report of six cases. Acta Cytol 26:488-490, 1982

2. Aron M, Kapila K, Sarkar C, Verma K: Microfilariae of Wuchereria bancrofti in cyst fluid of tumors of the brain: a report of three cases. Diagn Cytopathol 26:158-162, 2002

3. Carme B, Boulesteix J, Boutes H, Puruehnce MF: Five cases of encephalitis during treatment of loiasis with diethylcarbamazine. Am J Trop Med Hyg 44:684-690, 1991

4. Dange P, Goel G, Ghosh N, Agarwal S, Kaushal S, Jain S: Mammary filariasis masquerading as breast carcinoma. Breast J 20:549-551, 2014

5. Jain S, Desai P, Goel G, Singh N, Kaushal S: Urinary filariasis masquerading as the bladder tumor: a case report with cyto-histological correlation. J Cytol 32:124-126, 2015

6. Miller CE, Bain BJ: The utility of blood and bone marrow films and trephine biopsy sections in the diagnosis of parasitic infections. Mediterr J Hematol Infect Dis 7:e2015039, 2015

7. Naik PS, Tamboli SS, Agashe SR, Patil PP, Kale Y: Cheek swelling: an unusual presentation of filariasis. J Vector Borne Dis 52:270-272, 2015

8. Paliwal VK, Goel G, Vema R, Pradhan S, Gupta RK: Acute disseminated encephalomyelitis following filarial infection. J Neurol Neurosurg Psychiatry 83:347-349, 2012

9. Pittella JEH: Pathology of CNS parasitic infections. Handb Clin Neurol 114:65-88, 2013

10. Rivera-Zengotita M, Yachnis AT: Gliosis versus glioma?: don't grade until you know. Adv Anat Pathol 19:239-249, 2012

11. Singh M, Majumdar K, Shramana M, Ravindra S, Daljit S: Incidental detection of an isolated microfilaria in squash cytology of an oligodendroglioma: the unexpected 'worm' in the pie. Cytopathology 25:61-63, 2014

12. Şovrea AS, Boşca AB, Georgiu C, Constantin AM, Ben Abdalah MA, Gheban D: The diagnostic value of immunohistochemistry and silver impregnation techniques for characterization of normal, reactive and tumoral astrocytes. Rom J Morphol Embryol 55 (2 Suppl):525-538, 2014

\section{Disclosures}

The authors report no conflict of interest concerning the materials or methods used in this study or the findings specified in this paper.

\section{Author Contributions}

Conception and design: Shrivastava. Acquisition of data: Shrivastava, Arora, Khare, Goel. Analysis and interpretation of data: Shrivastava, Arora, Goel, Kapoor. Critically revising the article: Kapoor.

\section{Correspondence}

Adesh Shrivastava, Department of Neurosurgery, All India Institute of Medical Sciences, Saket Nagar, Bhopal (MP) 462024, India. email: dr.adesh.shrivastava@gmail.com. 